\title{
Differentiation of brain metastases from small and non-small lung cancers using apparent diffusion coefficient (ADC) maps
}

Sebastian Johannes Müller* (10, Eya Khadhraoui, Nicole E. Neef, Christian Heiner Riedel and Marielle Ernst

\begin{abstract}
Background: Brain metastases are particularly common in patients with small cell lung cancer (SCLC) and non-small cell lung cancer (NSCLC), with NSCLC showing a less aggressive clinical course and lower chemo- and radio sensitivity compared to SCLC. Early adequate therapy is highly desirable and depends on a reliable classification of tumor type. The apparent diffusion coefficient is a noninvasive neuroimaging marker with the potential to differentiate between major histological subtypes. Here we determine the sensitivity and specificity of the apparent diffusion coefficient to distinguish between NSCLC and SCLC.

Methods: We enrolled all NSCLC and SCLC patients diagnosed between 2008 and 2019 at the University Medical Center Göttingen. Cranial MR scans were visually inspected for brain metastases and the ratio of the apparent diffusion coefficient (ADC) was calculated by dividing the ADC measured within the solid part of a metastasis by a reference ADC extracted from an equivalent region in unaffected tissue on the contralateral hemisphere.

Results: Out of 411 enrolled patients, we detected 129 patients (83 NSCLC, 46 SCLC) with sufficiently large brain metastases with histologically classified lung cancer and no hemorrhage. We analyzed 185 brain metastases, 84 of SCLC and 101 of NSCLC. SCLC brain metastases showed an ADC ratio of $0.68 \pm 0.12$ SD, and NSCLC brain metastases showed an ADC ratio of $1.47 \pm 0.31$ SD. Receiver operating curve statistics differentiated brain metastases of NSCLC from SCLC with an area under the curve of 0.99 and a $95 \% \mathrm{Cl}$ of 0.98 to 1, $p<0.001$. Youden's J cut-point is 0.97 at a sensitivity of 0.989 and a specificity of 0.988 .
\end{abstract}

Conclusions: In patients with lung cancer and brain metastases with solid tumor parts, ADC ratio enables an ad hoc differentiation of SCLC and NSCLC, easily achieved during routine neuroradiological examination. Non-invasive MR imaging enables an early-individualized management of brain metastases from lung cancer.

Trial registration: The study was registered in the German Clinical Trials Register (DRKS00023016).

Keywords: Brain metastases, Diffusion, SCLC, NSCLC, ADC

*Correspondence: sebastian.mueller@med.uni-goettingen.de Department of Diagnostic and Interventional Neuroradiology, GeorgAugust-University Göttingen, Robert-Koch-Str. 40, 37075 Göttingen, Germany

\section{Background}

The incidence of brain metastases in patients with lung cancer is approximately $20 \%$ [1]. Histologically, $38 \%$ of patients suffer from small cell lung cancer (SCLC), and in $62 \%$ non-small cell lung cancer (NSCLC) is found [2].

A reliable radiological marker for differentiating these subtypes could accelerate the start of therapy. A fast differentiation is essential for an efficient therapy, 
especially in case of SCLC. Today, biopsy and histological workup are essential before therapy is started. Beside surgical risks, wound healing can be insufficient under systemic therapy [3].

The relationship between diffusion restricted brain metastasis and underlying pathology is still a matter of debate [4]. There are a number of heterogeneous studies including very few patients and dealing with the value of diffusion imaging in patients with brain metastases, many of which even attempt to find a correlation to certain gene expressions [5]. Statistically, in solid tumor parts more cell membranes should be included in $1 \mathrm{~cm}^{3}$ of a small cell carcinoma than in NSCLC, because the cytoplasmic diameter is smaller in SCLC [6]. Thus, we hypothesize that the diffusion of water molecules should be less restricted in NSCLS compared to SCLC. We analyzed whether a differentiation of SCLC and NSCLC in brain metastases can be achieved with diffusion weighted imaging (DWI), i.e. the apparent diffusion coefficient (ADC) mapping. First evidence for an ADC based differentiation of histological subtypes of brain metastases from lung cancer stems from a most recent study [7]. However, time-consuming offline analyses restrict the implementation of this approach into everyday clinical routine. Here, we evaluated a practically feasible procedure that can be implemented in clinical routine and promote timely clinical decisions.

\section{Methods}

\section{Study design}

This was a retrospective analysis of a single-center noninterventional observational study. We followed the STARD 2015 protocol [8]. Institutional review board approval was obtained. The study was registered in the German Clinical Trial Register (No. DRKS00023016).

\section{Participants}

We analyzed patients with pathologically confirmed SCLC or NSCLC and an MRI of the brain, regardless of the MRI manufacturer or the field strength. The study had to contain (1) a T1-weighted sequence with contrast agent (CA), (2) a T2-weighted sequence for detecting cystic parts and (3) a DWI Sequence with ADC mapping as calculated by the manufacturer.

The patients were checked, and excluded, if (1) no brain metastasis were found, (2) the size of the solid tumor parts were not large enough $\left(<0.25 \mathrm{~cm}^{2}\right)$ for measurements or (3) more than one tumor origin was known at time of MRI. Every patient was included only once, with the first cranial MRI containing at least one brain metastasis. Up to 10 metastases per patient were to be included. Brain metastases with large intra-tumoral hemorrhage ( $>50 \%$ of the solid tumor part) were excluded.

\section{Test methods}

A database search with the terms "cMRI" and (NSCLC" or "SCLC" or "lung cancer") was performed in our picture archiving and communication system (PACS) for patients scanned between 01/01/2009 and 01/01/2020. These patients were checked in our local tumor board for histological data and additional diseases.

\section{Image analysis}

For comparing supra- and infra-tentorial metastases and different field strengths we developed a simple procedure. We measured the solid tumor part and the contralateral white matter and divided both values to calculate an ADC ratio, as shown in Fig. 1. A similar approach was used in a publication from Jung et al. [5] for comparing supra- and infratentorial brain metastases.

Two neuroradiology fellows ( $>2$ years' experience in MRI diagnostics, blinded to clinical information) independently evaluated the cases and measured the ADC

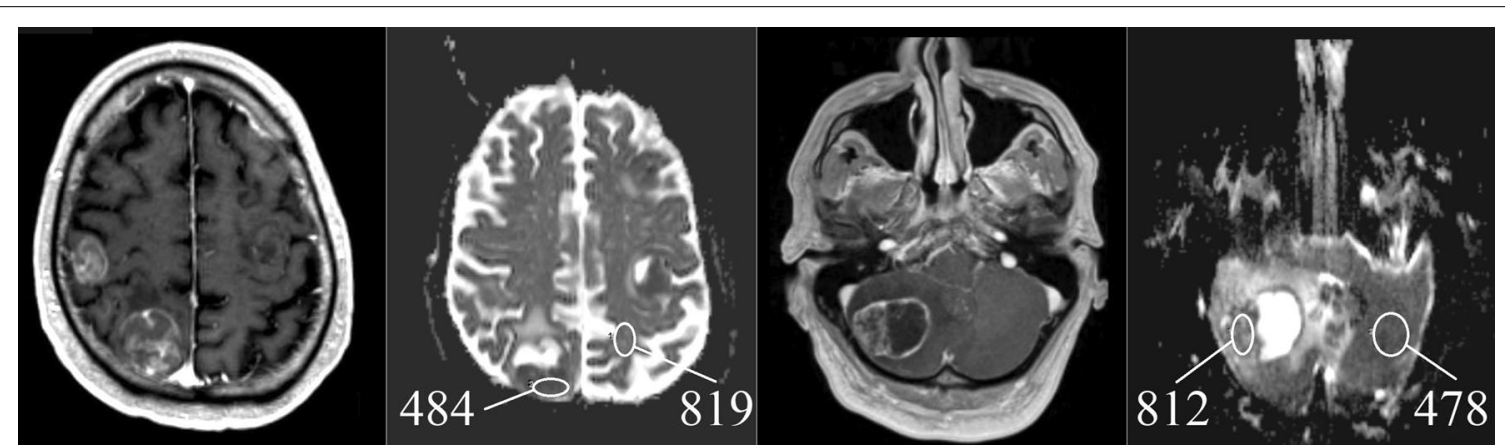

Fig. 1 Two examples of ADC-ratios of brain metastasis in 3-T MRI (T1 with contrast agent and ADC map): left: typical SCLC with ADC ratio of $484 / 819=0.59$, right: NSCLC with ADC ratio of $812 / 478=1.70$. SCLC small cell lung cancer, NSCLC non-small cell lung cancer, $A D C$ apparent diffusion coefficient 
values. Another third (blinded) neuroradiologist evaluated 30 randomly chosen sample cases. The measuring method was explained with five sample cases. No further training was necessary.

Centricity $^{\text {TM }}$ PACS RA1000 Workstation (GE Healthcare, Chicago, ILLINOIS, USA) was used for measurements. The maximum diameter of the solid tumor area was determined on the enhanced T1 images combined with T2 and ADC maps. Finally, the corresponding area recorded in the ADC map was measured. The region of interest (ROI) was manually selected by each neuroradiologist separately. Adjacent large blood vessels and cystic necrosis should be avoided. The average $A D C$ values was analyzed. In the event of a discrepancy (>15\% ADC ratio deviation), the case was discussed and examined for sources of error.

\section{Statistical analysis}

The program Statistica, version 13 (TIBCO Software Inc., Palo Alto, CALIFORNIA, USA) was used. Significance level was set to $P<0.05$. The comparison of ADC ratios of SCLC and NSCLC was performed using two sided Mann-Whitney U tests. Kruskal Wallis Test was used to compare ADC ratios of all different subtypes. Receiver Operating Curves (ROC) and Area Under Curve (AUC) values were used for calculating the ADC ratio with the highest diagnostic value to differentiate SCLC from NSCLC. The global optimum was determinated by maximizing Youden's J. To test inter-rater reliability we calculated interclass correlation coefficient (ICC) estimates and their 95\% confident intervals with $\mathrm{R}$ (packages irr, readxl, lpSolve and psych) based on mean-rating $(\mathrm{k}=3)$, absolute-agreement, and a 2 -way random-effects model.

\section{Results}

\section{Participants}

Our database contained 411 patients (162 females) with available imaging from 01.01.2009 to 01.01.2020. Mean age was $63.7 \pm 9.5$ years (mean \pm standard deviation, range 31-89 years). Patients with confirmed SCLC were on average $62.9 \pm 8.5$ (mean \pm standard deviation, range $40-83$ years, $n=110,40$ females) years old. Figure 2 shows a flowchart of the patients with included brain metastases in this study. Finally, 129 patients were included in the analysis. Five patients with intratumoral hemorrhage were excluded from the main analysis but measured and analyzed for comparison purposes. The distribution of histologies did not differ significantly and is demonstrated in Fig. 3a, d.

\section{Interrater agreement}

Inter rater reliability was good to excellent with an ICC estimate of 0.898 and a $95 \%$ confidence interval of 0.825 to 0.945 [9].

\section{MR Scanner and sequences}

MRI data of six different MR scanners were included. ADC maps were based on echo-planar imaging DWI (EPI-DWI) in all cases, mostly with a slice thickness of $4 \mathrm{~mm}$. Hence, ADC ratio is normalized and manufacturer and sequence independent, we abstain from a sequence/manufacturer specific evaluation, especially since our evaluated MRI were mainly performed on Siemens scanners, as shown in Table 1.

\section{Test results}

$S C L C$

In 46 of 110 patients (32 of them without/before radiation or chemotherapy) with histologically confirmed small cell lung cancer 84 measurable brain metastases were detected. Initial diagnoses were made in 17 patients by brain biopsy, and in 29 patients via lung biopsy. $1.82 \pm 1.16$ metastases per patient (mean $\pm S D$, range 1-6, median 1); in total 55 pre-therapeutic and 29 post-therapeutic brain metastases of patients with SCLC. Morphology of all SCLC metastases showed a distribution of $22 \%$ solid, $52 \%$ mixed cystic-solid and $26 \%$ cystic metastases.

Locations of metastases were in $11 \%$ of the patients only in the infratentorial and in $37 \%$ only in the supratentorial compartment. $52 \%$ of SCLC metastases were detected in both locations.

\section{NSCLC}

Sufficiently large brain metastases were found in 80 of 301 patients with confirmed NSCLC (73 of them without/before radiation or system therapy). First diagnoses were achieved in 21 patients by direct biopsy/resection of brain metastases, and in 59 cases by lung biopsy. Histology revealed in 66 cases adenocarcinomas (AC), and in 14 squamous cell carcinomas (SCC). Overall 93 NSCLC metastases $(1.19 \pm 0.44$ metastases per patient, range $1-3$, median 1 ), a total of 71 pre-therapeutic and 22 post-therapeutic values, were analyzed. Morphology contained $37 \%$ solid, $40 \%$ mixed cystic-solid and $23 \%$ cystic metastases.

In $12 \%$ (AC $12 \%$, SCC $14 \%$ ) of the patients only the infratentorial and in 49\% (AC 45\%, SCC 64\%) only the supratentorial compartment was affected. In 39\% (AC 


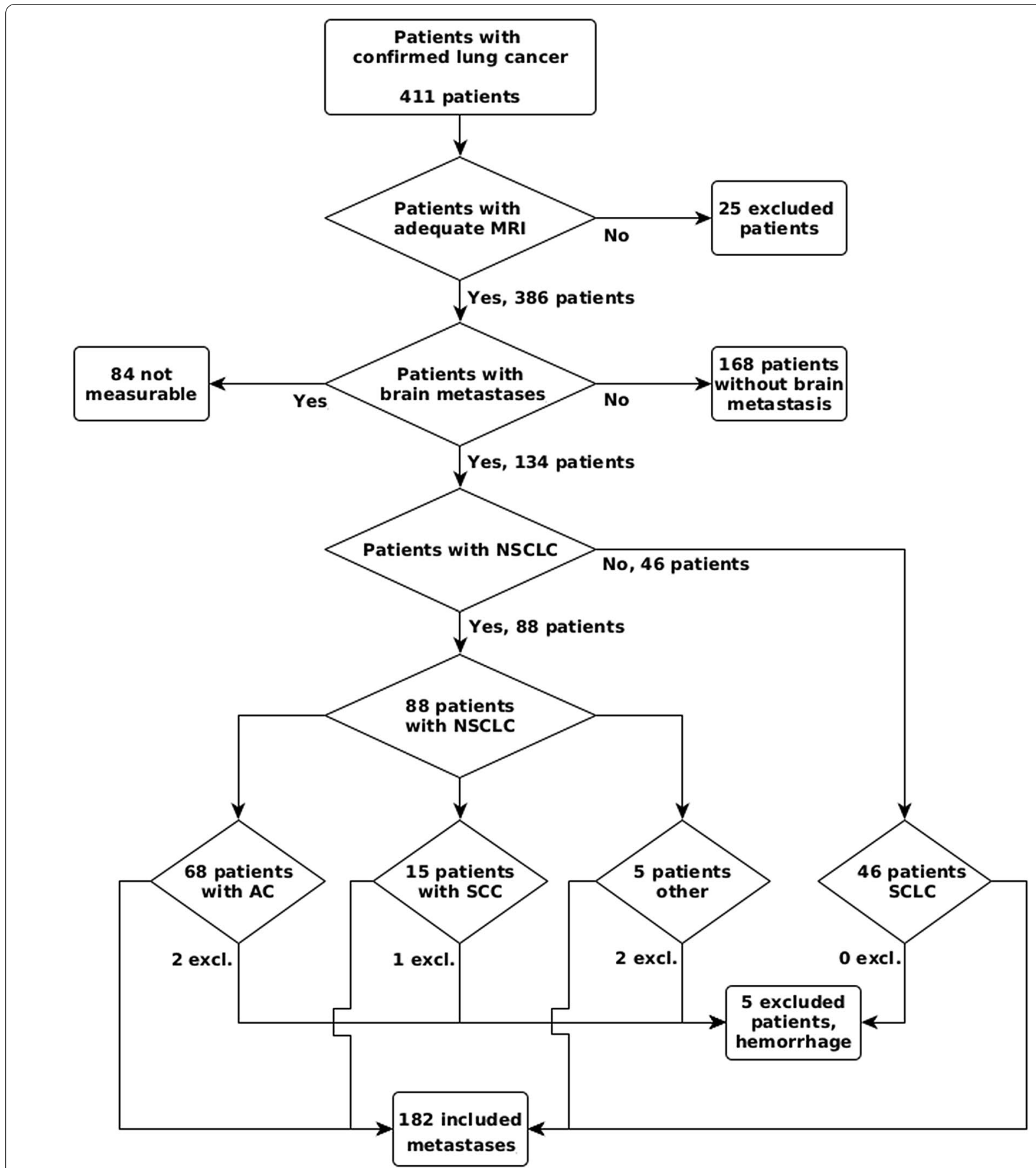

Fig. 2 Participant flow chart. AC adenocarcinoma, ADC apparent diffusion coefficient, CA contrast agent, NSCLC non-small cell lung cancer, SCC squamous cell carcinoma, SCLC small cell lung cancer

43\%, SCC 22\%) NSCLC metastases were detected in both locations.

\section{Other}

Three patients had neither AC nor SCC, i.e. two had large cell neuroendocrine tumor, and one had signet ring cell carcinoma. Five patients with hemorrhage of brain metastases were separately analyzed.

\section{Differentiation of SCLC and NSCLC}

The mean ADC ratios of brain metastasis were $0.68 \pm 0.12$ for SCLC $(n=84)$, and $1.47 \pm 0.31$ for 

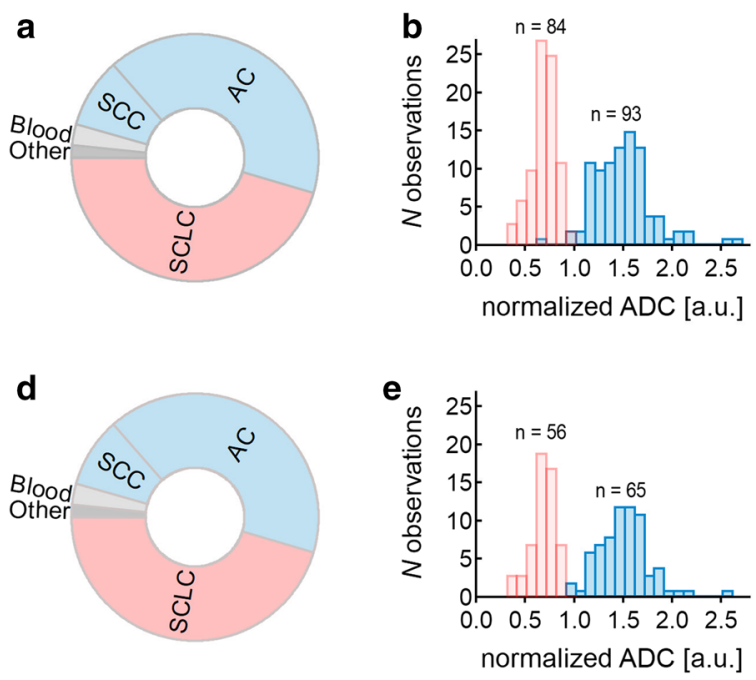
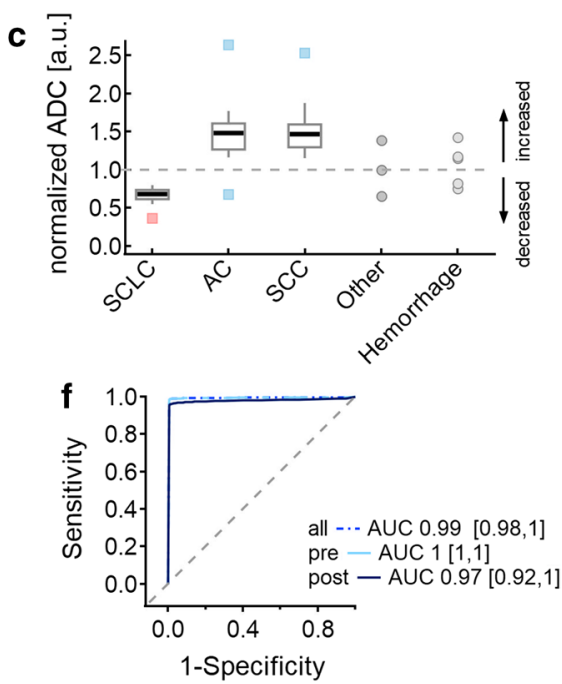

Fig. 3 Overview results - a Pie charts of the patient cohort: distribution of patients with measurable brain metastases of lung cancer, and $\mathbf{d}$ distribution of only pre-therapeutic patients. $\mathbf{b}$ Histogram: distribution of ADC ratio of all measured metastases and, e only pre-therapeutic metastases of small cell lung cancer (left) and non-small cell lung cancer (right, contains only squamous cell carcinoma and adenocarcinoma). c Whisker-Box-Plot of ADC ratios grouped by histologies. $\mathbf{f}$ Receiver operating characteristics curve and area und curve of all, pre- and post-therapeutic patients with confidence interval. AC adenocarcinoma, SCC squamous cell carcinoma, SCLC small cell lung cancer

Table 1 MR scanners

\begin{tabular}{|c|c|c|c|c|c|c|}
\hline Scanner & $N$ (percent) & $\mathrm{SCLC}$ & $A C$ & SCC & Other & Blood \\
\hline 3T-TIM Trio ${ }^{1}$ & $123(66)$ & 54 & 49 & 15 & 1 & 4 \\
\hline 1.5T-AvantoFit ${ }^{1}$ & $41(22)$ & 20 & 18 & 1 & 1 & 1 \\
\hline 3T-PrismaFit ${ }^{1}$ & $11(6)$ & 4 & 6 & 0 & 1 & - \\
\hline 1.5T-Sonata ${ }^{1}$ & $6(3)$ & 3 & 2 & 1 & - & - \\
\hline 3T-Discovery² & $3(2)$ & 3 & - & - & - & - \\
\hline 1.5T-Intera ${ }^{3}$ & $1(1)$ & - & 1 & - & - & - \\
\hline
\end{tabular}

\footnotetext{
1 Siemens

${ }^{2}$ GE Healthcare

${ }^{3}$ Philips
}

NSCLC. A histogram of the distribution of ADC ratios of all measured metastases is shown in Fig. 3b.

The two sided Mann-Whitney U tests showed a significant differentiation between SCLC and NSCLC $(p<0.001)$. Youden's J analyses of AUC estimated ADC ratio $<0.97$ being the optimal cut-off value with a sensitivity of $99 \%(83 / 84)$ and specificity of $99 \%$ (92/93) for detecting SCLC, as shown in Fig. 3f. An AD ratio of 0.98 shows a sensitivity of $100 \%$ and specificity of $97 \%(90 / 93)$. Figure 3c demonstrates a box-plot of the four subgroups and the excluded intratumoral hemorrhage.

\section{Pre-therapeutic differentiation of SCLC and NSCL}

In $100 \%$ of the pre-therapeutic SCLC cases, the ADC ratio was 0.9 or below $(0.66 \pm 0.12$ for the solid part of the metastasis). ADC ratio (NSCLC) was $1.50 \pm 0.30$. ADC ratios between 0.89 and 0.96 showed a sensitivity and specificity of $100 \%$ in detecting SCLC $(n=55)$ versus NSCLC ( $\mathrm{n}=71,58 \mathrm{AC}$ and $13 \mathrm{SCC})$; pre-therapeutic, without hemorrhage and with either AC or SCC histology. Only one (in this analysis excluded) pre-therapeutic not-SCLC metastasis (of a large cell neuroendocrine tumor) showed an ADC ratio $<0.9$. Figure $3 \mathrm{e}$ demonstrates the distribution of measured ADC ratios in a histogram. The two sided Mann-Whitney U tests stated a significant differentiation between SCLC and NSCLC $(p<0.001)$. 


\section{Post-therapeutic ADC ratio}

Post-therapeutic brain metastases (29 SCLC, 18 AC, 4. SCC) showed an average ADC ratio of $0.70 \pm 0.13$ for SCLC, and $1.37 \pm 0.34$ for NSCLC. The two sided MannWhitney $\mathrm{U}$ tests still showed a significant differentiation between SCLC and NSCLC $(p<0.001)$. RoC and AuC analyses estimated $\mathrm{ADC}$ ratio $<0.97$ being the optimal cut-off value with a sensitivity of $100 \%$ and specificity of 96\% (23/24) for detecting SCLC.

\section{Subgroup analysis}

Separate analysis for AC and SCC demonstrated slightly smaller ADC ratios for AC but no significant differences, as demonstrated in Table 2. Two-tailed Kruskal Wallis Test of the four different subtypes and intratumoral hemorrhage showed significant differences between (1) SCLC and AC and (2) SCLC and SCC, but no significant differences between the other groups, as shown in Additional file 2: Table S1.

\section{Discussion}

Our study in 411 patients with lung cancer demonstrates the importance of pre-therapeutic cranial MRI, which is still a matter of debate [10, 11], even if neurologic metastases [12] are common in lung cancer. Following the results of our study, we can distinguish SCLC from NSCLC on initial diagnostic work-up.

Although brain metastases are improbable in early stage NSCLC without central neurologic symptoms [13], the risk of brain metastasis, especially in SCLC is omnipresent [14]. Large studies show bad survival rates for patients with limited [15] and extended disease [16-18]. Even if new system therapies have increased to mean survival time compared to old studies [19], an early and effective therapy seems to be an important factor in limited disease $[20,21]$. Hence, even in extended diseases,

Table 2 ADC ratio subtype analyses of brain metastases

\begin{tabular}{llllll}
\hline Subtype & $N$ & Mean & Min & Max & SD \\
\hline SCLC & 84 & 0.68 & 0.36 & 0.97 & 0.12 \\
Pre-therapeutic & 55 & 0.66 & 0.36 & 0.88 & 0.12 \\
Post-therapeutic & 29 & 0.70 & 0.47 & 0.97 & 0.13 \\
AC & 76 & 1.47 & 0.68 & 2.64 & 0.30 \\
Pre-therapeutic & 58 & 1.50 & 1.00 & 2.64 & 0.28 \\
Post-therapeutic & 18 & 1.37 & 0.68 & 2.15 & 0.36 \\
SCC & 17 & 1.52 & 0.97 & 2.53 & 0.36 \\
Pre-therapeutic & 13 & 1.56 & 0.97 & 2.53 & 0.39 \\
Post-therapeutic & 4 & 1.38 & 1.10 & 1.66 & 0.25 \\
Other & 3 & 1.01 & 0.65 & 1.39 & 0.37 \\
Hemorrhage & 5 & 1.06 & 0.75 & 1.42 & 0.28 \\
\hline SCLC smallinn
\end{tabular}

SCLC small cell lung cancer, $A C$ adenocarcinoma, SCC squamous cell carcinoma fast diagnosis and therapy is important [22-24]. The prognosis remains poor, although the combination of chemotherapy with new immune therapies seems to improve the survival $[25,26]$. In contrast to SCLC the more common AC [27] and SCC [28] show a slightly bettering of the long time survival rate.

Thus an additional surgery can influence the course of the disease [29] as well as a complicated wound healing [3]. Radiosurgery and whole brain therapy are possible alternatives to surgery [30,31].

"Nomen est omen", but it has long been known that the average cytoplasmic diameter of SCLC is significantly lower than NSCLC $(p<0.0001)$, with approx. 8.4 microns versus 15 microns, as shown by Vollmer [6]. A small study from Korea could not show a significant lower diffusion restriction using an ADC-ratio-like approach, but a correlation with epidermal growth factor receptor (EGFR) expression [5]. A recent study from Turkey showed a possible differentiation of SCLC and AC using ADC-Histograms [7]. Compared to other studies more patients with SCLC and less with SCC were included in our study. The mean ADC ratio of SCC and AC was higher than in other studies and the differentiation between SCLC and NSCLC was clearer. ADC ratios of tumor cells are multifactorial influenced, e.g. by irregular angiogenesis or abnormal extracellular matrix [32, 33], and did not only depend on cell diameters. Although the sensitivity and specificity in our study seems to be astonishing high. Mainly, because the low cell diameter seems to be the dominating factor in pre-therapeutic SCLC, which leads to a lowering of the ADC.

Localization and morphology of brain metastases were similar to other studies $[34,35]$.

The occurrence of brain metastases in our cohort was significantly higher $(56 \%$ at all, for pre-therapeutic patients with (measureable) brain metastases: $31 \%$ SCLC, $25 \%$ NSCLC, respectively) than in other studies [1,36]. We only included patients with neuroradiologic imaging, which leads bias towards potentially neurologic strongly affected patients with a higher probability of brain metastases.

\section{Limitations}

Measurement of solid tumor parts of metastases keeps a challenging task. The wrong selection of tumor edema, cystic parts or blood can strongly influence the ADC ratio. For this reason, we separately controlled the measurements by two neuroradiology fellows. A third (blinded) neuroradiologist repeated the measurement for 30 randomly chosen cases, and again a good to excellent interrater reliability was demonstrated.

Visual features of metastasis allow a clear differentiation of the solid tumor portion, if enhanced T1-, T2- and 
DWI sequences are viewed in parallel, each with a sufficient layer thickness $(\leq 4 \mathrm{~mm})$ and with the same orientation (e.g. transversal).

Even if in cases with heterogeneous solid tumor parts small differences in the measurements were noticed, the inter-rater-reliability was sufficient. Nevertheless, an objective procedure for selecting the region of interest should be evaluated, which is needed to establish this method into clinical routine.

The count of post-therapeutic measurable metastases was low, wherefore we did not perform a subgroup analysis for chemotherapy and radiotherapy. Although, we noticed a greater variance in the measured values.

\section{Outlook}

Prospective data should be collected and analyzed to confirm our findings. The extent of resection of a metastasis beyond the incision margins is still unclear, here DWI sequences can reveal additional information [37]. Metastases after radiation and/or chemotherapy showed an different ADC ratio, which may be used for therapy monitoring [38-40].

\section{Conclusion}

In pre-therapeutic patients with lung cancer and brain metastases with solid tumor parts, ADC ratio enables an excellent differentiation of SCLC and NSCLC.

\begin{abstract}
Abbreviations
AC: Adeno carcinoma; ADC: Apparent diffusion coefficient; AUC: Area under curve; CMRI: Cranial magnetic resonance imaging; DWI: Diffusion weighted imaging; ICC: Interclass correlation coefficient; MR: Magnetic resonance; NSCLC: Non-small cell lung cancer; ROC: Receiver operating characteristics: SCC: Squamous cell carcinoma; SCLC: Small cell lung cancer; SD: Standard deviation.
\end{abstract}

\section{Supplementary Information}

The online version contains supplementary material available at https://doi. org/10.1186/s12880-021-00602-7.

Additional file 1. STARD 2015 protocol.

Additional file 2. Kruskal Wallis Test results for the differentiation of lung cancer subtypes using ADC-ratio.

\section{Acknowledgements}

None.

\section{Authors' contributions}

All authors have reviewed and approved the submitted manuscript for publication. SM was the project administrator and organized the data curation, measurements, design, literature review, and writing of the manuscript. EK contributed to data collection, measurements and analysis. ME contributed to measurements, analysis, literature review, and edited the manuscript. $\mathrm{CH}$ contributed to the conceptualization, literature review, and design of the study. NN contributed to the formal analysis and literature review of the study, draft, review, and edited the manuscript. The authors agree to be accountable for all aspects of work ensuring integrity and accuracy. All authors read and approved the final manuscript.

\section{Funding}

Open Access funding enabled and organized by Projekt DEAL. No funding was received for the conduct of this study. The authors acknowledge support for the publication fees by the German Research Foundation and the Open Access Publication Funds of the Göttingen University.

\section{Availability of data and materials}

The datasets used and analyzed during the current study are available from the corresponding author on reasonable request.

\section{Declarations}

\section{Ethical approval and consent to participate}

The study was ethically approved by the institutional review board. ("Ethikkommission der Universitätsmedizin Göttingen", Göttingen, Germany, No. 08/05/20) and registered in the German Clinical Trials Register (DRKS00023016). The full study protocol is provided in the supplemental data, see Additional file 1: STARD 2015 checklist. This retrospective study adhered to the 2013 Helsinki Declaration. The approach and preliminary results (abstract only) were presented at the Proceedings of the 55th Annual Conference of the German Society for Neuroradiology [41].

\section{Consent for publication}

Not applicable.

\section{Competing interests}

The authors declare that they have no competing interests.

Received: 6 December 2020 Accepted: 5 April 2021

Published online: 15 April 2021

\section{References}

1. Barnholtz-Sloan JS, Sloan AE, Davis FG, Vigneau FD, Lai P, Sawaya RE. Incidence proportions of brain metastases in patients diagnosed (1973 to 2001) in the metropolitan detroit cancer surveillance system. J Clin Oncol. 2004;22:2865-72.

2. Nussbaum ES, Djalilian HR, Cho KH, Hall WA. Brain metastases: histology, multiplicity, surgery, and survival. Cancer. 1996;78:1781-8.

3. Beyene RT, Derryberry SL, Barbul A. The effect of comorbidities on wound healing. Surg Clin N Am. 2020;100:695-705.

4. Duygulu G, Ovali GY, Çalli C, Kitis Ö, Yünten N, Akalin T, et al. Intracerebral metastasis showing restricted diffusion: correlation with histopathologic findings. Eur J Radiol. 2010;74:117-20.

5. Jung WS, Park CH, Hong C-K, Suh SH, Ahn SJ. Diffusion-weighted imaging of brain metastasis from lung cancer: correlation of MRI parameters with the histologic type and gene mutation status. Am J Neuroradiol. 2018;39:273-9.

6. Vollmer RT. The effect of cell size on the pathologic diagnosis of small and large cell carcinomas of the lung. Cancer. 1982;50:1380-3.

7. Bozdağ $M, E r$, Çinkooğlu A. Histogram analysis of ADC maps for differentiating brain metastases from different histological types of lung cancers. Can Assoc Radiol J. 2020.https://doi.org/10.1177/0846537120 933837.

8. Bossuyt PM, Reitsma JB, Bruns DE, Gatsonis CA, Glasziou PP, Irwig L, et al. STARD 2015: an updated list of essential items for reporting diagnostic accuracy studies. Radiology. 2015;277:826-32.

9. Koo TK, Li MY. A guideline of selecting and reporting intraclass correlation coefficients for reliability research. J Chiropr Med. 2016;15:155-63.

10. Hudson Z, Internullo E, Edey A, Laurence I, Bianchi D, Addeo A. Brain imaging before primary lung cancer resection: a controversial topic. ecancermedicalscience. 2017. https://doi.org/10.3332/ecancer.2017.749.

11. Balekian AA, Fisher JM, Gould MK. Brain imaging for staging of patients with clinical stage IA non-small cell lung cancer in the national lung screening trial. Chest. 2016;149:943-50. 
12. Wanleenuwat $P$, Iwanowski P. Metastases to the central nervous system: molecular basis and clinical considerations. J Neurol Sci. 2020;412:116755

13. Wasp GT, Del Prete C, Farrell JAD, Dragnev KH, Russo G, Atkins GT, et al. Impact of neuroimaging in the pretreatment evaluation of early stage non-small cell lung cancer. Heliyon. 2020;6:e04319.

14. Pezzi TA, Fang P, Gjyshi O, Feng L, Liu S, Komaki R, et al. Rates of overall survival and intracranial control in the magnetic resonance imaging era for patients with limited-stage small cell lung cancer with and without prophylactic cranial irradiation. JAMA Netw Open. 2020;3:e201929.

15. Farrell MJ, Yahya JB, Degnin C, Chen Y, Holland JM, Henderson MA, et al. Prophylactic cranial irradiation for limited-stage small-cell lung cancer: survey of US radiation oncologists on current practice patterns. Clin Lung Cancer. 2018;19:371-6.

16. Minna JD, Bunn PA, Carney DN, Cohen MH, Cuttita F, Fosieck BE, et al. Experience of the National Cancer Institute (USA) in the treatment and biology of small cell lung cancer. Bull Cancer (Paris). 1982;69:83-93.

17. Chung J-H, Kang SY, Wu H-G, Seo YS, Kim D-W, Kang KW, et al. Risk stratification of symptomatic brain metastases by clinical and FDG PET parameters for selective use of prophylactic cranial irradiation in patients with extensive disease of small cell lung cancer. Radiother Oncol. 2020;143:81-7.

18. Cramer-van der Welle CM, Schramel FMNH, Leeuwen AS, Groen HJM, Garde EMW, for the Santeon SCLC Study Group, et al. Real-world treatment patterns and outcomes of patients with extensive disease small cell lung cancer. Eur J Cancer Care (Engl). 2020. https://doi.org/10.1111/ecc. 13250.

19. Campobasso O, Invernizzi B, Musso M, Berrino F. Survival rates of lung cancer according to histological type. Br J Cancer. 1974;29:240-6.

20. Glatzer M, Rittmeyer A, Müller J, Opitz I, Papachristofilou A, Psallidas I, et al. Treatment of limited disease small cell lung cancer: the multidisciplinary team. Eur Respir J. 2017:50:1700422.

21. Higgins KA, Gorgens S, Sudmeier LJ, Faivre-Finn C. Recent developments in limited stage small cell lung cancer. Transl Lung Cancer Res. 2019;8:S147-52.

22. Allen J, Jahanzeb M. Extensive-stage small-cell lung cancer: evolution of systemic therapy and future directions. Clin Lung Cancer. 2008;9:262-70.

23. Oronsky B, Reid TR, Oronsky A, Carter CA. What's new in SCLC? A review. Neoplasia. 2017;19:842-7.

24. Nesbit EG, Leal TA, Kruser TJ. What is the role of radiotherapy for extensive-stage small cell lung cancer in the immunotherapy era? Trans Lung Cancer Res. 2019;8:S153-62.

25. Kahnert K, Kauffmann-Guerrero D, Huber RM. SCLC-state of the art and what does the future have in store? Clin Lung Cancer. 2016;17:325-33.

26. Calles A, Aguado G, Sandoval C, Álvarez R. The role of immunotherapy in small cell lung cancer. Clin Transl Oncol. 2019;21:961-76.

27. Travis WD, Brambilla E, Noguchi M, Nicholson AG, Geisinger KR, Yatabe Y, et al. International association for the study of lung cancer/American thoracic society/European respiratory society international multidisciplinary classification of lung adenocarcinoma. J Thorac Oncol. 2011;6:244-85.

28. Lee $H$, Jeong SH, Jeong B-H, Park HY, Lee K-J, Um S-W, et al. Incidence of brain metastasis at the initial diagnosis of lung squamous cell carcinoma on the basis of stage, excluding brain metastasis. J Thorac Oncol. 2016;11:426-31.

29. Alieva M, van Rheenen J, Broekman MLD. Potential impact of invasive surgical procedures on primary tumor growth and metastasis. Clin Exp Metastasis. 2018;35:319-31.

30. Rusthoven CG, Yamamoto M, Bernhardt D, Smith DE, Gao D, Serizawa $T$, et al. Evaluation of first-line radiosurgery vs whole-brain radiotherapy for small cell lung cancer brain metastases: the FIRE-SCLC cohort study. JAMA Oncol. 2020:6:1028.

31. Chang EL, Hassenbusch SJ, Shiu AS, Lang FF, Allen PK, Sawaya R, et al. The role of tumor size in the radiosurgical management of patients with ambiguous brain metastases. Neurosurgery. 2003;53:272-81.

32. Guo S, Martin MG, Tian C, Cui J, Wang L, Wu S, et al. Evaluation of detection methods and values of circulating vascular endothelial growth factor in lung cancer. J Cancer. 2018;9:1287-300.

33. Reuten R, Zendehroud S, Nicolau M, Fleischhauer L, Laitala A, Kiderlen S, et al. Basement membrane stiffness determines metastases formation. Nat Mater. 2021. https://doi.org/10.1038/s41563-020-00894-0.

34. Wang G, Xu J, Qi Y, Xiu J, Li R, Han M. Distribution of brain metastasis from lung cancer. Cancer Manag Res. 2019;11:9331-8.

35. Takano K, Kinoshita M, Takagaki M, Sakai M, Tateishi S, Achiha T, et al. Different spatial distributions of brain metastases from lung cancer by histological subtype and mutation status of epidermal growth factor receptor. Neuro-Oncol. 2016;18:716-24.

36. Yang B, Lee H, Um S-W, Kim K, Zo Jl, Shim YM, et al. Incidence of brain metastasis in lung adenocarcinoma at initial diagnosis on the basis of stage and genetic alterations. Lung Cancer. 2019;129:28-34.

37. Zakaria R, Das K, Radon M, Bhojak M, Rudland PR, Sluming V, et al. Diffusion-weighted MRI characteristics of the cerebral metastasis to brain boundary predicts patient outcomes. BMC Med Imaging. 2014. https:// doi.org/10.1186/1471-2342-14-26.

38. Borghei-Razavi H, Sharma M, Emch T, Krivosheya D, Lee B, Muhsen B, et al. Pathologic correlation of cellular imaging using apparent diffusion coefficient quantification in patients with brain metastases after gamma knife radiosurgery. World Neurosurg. 2020;134:e903-12.

39. Liu Y, Liu X, Xu L, Liu L, Sun Y, Li M, et al. Magnetic resonance imaging evaluation of treatment efficacy and prognosis for brain metastases in lung cancer patients after radiotherapy: a preliminary study: MRI evaluation after radiotherapy in BM. Thorac Cancer. 2018;9:865-73.

40. Liu K, Ma Z, Feng L. Apparent diffusion coefficient as an effective index for the therapeutic efficiency of brain chemoradiotherapy for brain metastases from lung cancer. BMC Med Imaging. 2018. https://doi.org/ 10.1186/s12880-018-0275-3

41. Müller SJ, Khadhraoui E, Riedel CH. 55. Jahrestagung der Deutschen Gesellschaft für Neuroradiologie e.V. Clin Neuroradiol. 2020;30:136.https://doi.org/10.1007/s00062-020-00951-w.

\section{Publisher's Note}

Springer Nature remains neutral with regard to jurisdictional claims in published maps and institutional affiliations.
Ready to submit your research? Choose BMC and benefit from:

- fast, convenient online submission

- thorough peer review by experienced researchers in your field

- rapid publication on acceptance

- support for research data, including large and complex data types

- gold Open Access which fosters wider collaboration and increased citations

- maximum visibility for your research: over 100M website views per year

At BMC, research is always in progress.

Learn more biomedcentral.com/submissions 